\title{
Respiratory-chain deficiency presenting as diffuse mesangial sclerosis with NPHS3 mutation
}

\author{
Esra Baskin • Umut Selda Bayrakci • Füsun Alehan • \\ Handan Ozdemir • Ayse Oner • Rita Horvath • \\ Virginia Vega-Warner • Friedhelm Hildebrandt • \\ Fatih Ozaltin
}

Received: 7 August 2010 /Revised: 31 December 2010 /Accepted: 4 January 2011 / Published online: 2 March 2011

(C) IPNA 2011

\begin{abstract}
Renal manifestations of mitochondrial cytopathies have been described, but nephrotic syndrome with respiratory-chain disorders have been described extremely rarely. We report a 9-month-old boy with a mitochondrial cytopathy preceded by a 2-month history of steroid-resistant nephrotic syndrome. Percutaneous renal biopsy revealed diffuse mesangial sclerosis, and mutational analysis was compatible with PLCE1 mutation. However, electron microscopic findings of renal tissue, sensorineural hearing loss,
\end{abstract}

E. Baskin $(\bowtie) \cdot$ U. Selda Bayrakci

Department of Pediatric Nephrology, Baskent University, Ankara, Turkey

e-mail: esrabaskin@yahoo.com

F. Alehan

Department of Pediatric Neurology, Baskent University,

Ankara, Turkey

H. Ozdemir

Department of Pathology, Baskent University,

Ankara, Turkey

A. Oner

Department of Pediatric Nephrology,

Dr. Sami Ulus Children Hospital,

Ankara, Turkey

R. Horvath

Department of Neurology, Mitochondrial Research Group, NewCastle, UK

V. Vega-Warner $\cdot$ F. Hildebrandt

Departments of Pediatrics and of Human Genetics,

University of Michigan,

Ann Arbor, MI, USA

F. Ozaltin

Department of Pediatric Nephrology, Hacettepe University,

Ankara, Turkey and other ocular and neurologic findings led us to suspect mitochondrial cytopathy. Muscle tissue analysis showed a deficiency of the respiratory chain complex IV. The clinical presentation of our patient is not typical for primary cytochrome oxidase (COX) deficiency but showed similarities with patients carrying AR mutations in COX10. This was the first case in the literature with both PLCE1 mutation and COX deficiency. We could not identify pathogenic mutations in the COX10 gene, suggesting that PLCE1 deficiency could be the cause of the secondary deficiency of COX. Another, more likely, possibility is that the mitochondriopathy phenotype is caused by another mutation homozygous by descent in a yet unidentified recessive gene.

Keywords Nephrotic syndrome · Mitochondriopathy Respiratory-chain complex · Diffuse mesangial sclerosis . PLCE1

\section{Introduction}

Diffuse mesangial sclerosis (DMS) is a histologically distinct variant of nephrotic syndrome (NS) characterized by early onset and progression to end-stage renal disease (ESRD) [1]. Recently, recessive mutations in the PLCE1(NPHS3) gene encoding phospholipase $\mathrm{C}$ epsilon 1 (PLCE1) have been described as a novel cause of idiopathic DMS [1,2]. Genetic defects of oxidative phosphorylation have been reported in congenital as well as late-onset NS in children and adults with multiple organ involvement [3-6]. To date, however, respiratory chain $(\mathrm{RC})$ deficiency has never been described in association with PLCE1 mutation in DMS. Here we report mitochondrial RC complex IV (cytochrome $\mathrm{C}$ oxidase, COX) deficiency in a 9-month-old boy presenting with DMS carrying pathogenic mutation in PLCE1. 


\section{Case report}

A 9-month-old boy (A1391-21) presented with a history of edema in his face, legs, and scrotum of 2 months' duration on admission. He was diagnosed with NS in a local hospital and treated with intravenously administered human albumin and orally administered methylprednisolone $(2 \mathrm{mg} / \mathrm{kg}$ day) for 4 weeks. He was referred to our hospital after 1 month of follow-up due to unresponsiveness to steroid treatment and poor clinical condition. The child was born full term after an uneventful pregnancy to healthy, first-degreerelated parents. His history during the postnatal and early infancy period was uneventful, with normal growth and development until the onset of NS. There was no history of NS or any other renal disease in his family. On admission, physical examination revealed a height of $70 \mathrm{~cm}(25-50$ th centile for age), weight $10 \mathrm{~kg}$ (75-90th centile for age) and head circumference $46 \mathrm{~cm}$ (50th centile for age). Blood pressure, heart rate, and respiratory rate were $134 / 82 \mathrm{mmHg}$, $132 / \mathrm{min}$, and $34 / \mathrm{min}$, respectively. No dysmorphic appearance was noted. His abdomen was distended because of ascites, and there was $2+$ pretibial edema. The patient had an axial hypotonicity, and funduscopic examination revealed salt-and-pepper-like appearance in the retina, with irregular yellow pigmentation of the fovea. Laboratory evaluation showed significant hypoproteinemia (3.3 g/dl), hypoalbuminemia $(1.8 \mathrm{~g} / \mathrm{dl})$, and significant proteinuria (protein/creatinine: $46.2 \mathrm{mg} / \mathrm{mg}$ creatinine) with high triglyceride $(528 \mathrm{mg} / \mathrm{dl})$ and cholesterol $(699 \mathrm{mg} / \mathrm{dl})$ levels. Blood urea nitrogen (BUN) and creatinine levels were $114 \mathrm{mg} / \mathrm{dl}$ and $1.43 \mathrm{mg} / \mathrm{dl}$, respectively, with a glomerular filtration rate of $22 \mathrm{ml} / \mathrm{min}$ that was estimated by Schwartz formula. Complement 3 and 4 levels were $78 \mathrm{IU} / \mathrm{ml}$ (normal 75-80) and 16.4 IU/ml (normal 15-42), respectively. Antinuclear antibody and antibody to double-stranded DNA (dsDNA) were negative, and there was no evidence of previous hepatitis or intrauterine infections. Serology for rubella, toxoplasmosis, syphilis, and herpes simplex were also negative in both patient and mother.

Daily albumin infusions with furosemide were started. He remained nephrotic despite pulse methylprednisolone, cyclosporine A, and oral steroid treatment. A percutaneous renal biopsy was undertaken, and histological evaluation revealed renal cortical and medullar tissue with 21 glomeruli. Glomeruli showed irregular thickening and wrinkling of glomerular basal membranes, diffuse increase in mesangial matrix, and swollen endothelial cells. Mesangial matrix was markedly increased, extending into and narrowing capillary lumina. Some glomeruli show segmental sclerosis, and most showed solidification or global sclerosis. Shrunken and sclerotic glomeruli were surrounded by coronas of partly hypertrophied vacuolized podocytes (Fig. 1).
Focal tubular atrophy and focal fibrosis with minimal mononuclear inflammatory-cell infiltration was observed in the interstitium. Vacuolar degeneration of the cytoplasm of tubular cells and lipid droplets was also observed in most tubules. The muscular layer of the arteries and arterioles was thickened and associated with intimal proliferation and marked deposition of lipids in the walls. One afferent arterial and a few capillaries of two glomeruli showed hyaline lesions and necrosis. Variable amounts of segmental immunoglobulin M (IgM) and C3 deposition was observed in the mesangium and along peripheral capillary membrane. Electron-microscopic examination revealed irregular thickening and wrinkling of glomerular basal membranes. One glomerulus showed solidification and podocyte crowning. In other glomeruli, mesangial matrix was markedly increased, extending into and narrowing capillary lumina; endothelial cells were swollen, and podocytes were hypertrophied. Significant foot process effacement was noted. Tubular basement membrane thickening and irregularity were observed. Cytoplasm of tubules showed mitochondrial alterations such as unusual shapes and unusual patterns of cristae. In addition, various nonmitochondrial changes, such as cytoplasmic myelin-like inclusions, were noted. Clinical and pathological findings revealed the diagnosis of DMS. Serology for viral infections including cytomegalovirus, Ebstein-Barr virus, and Parvovirus were negative. Karyotype analysis showed $46 \mathrm{XY}$ with male phenotype. Direct exon sequencing for the PLCE1 gene revealed a homozygous TT deletion at position 5410-5411 in exon 3, causing a frameshift between amino acids 1804-1819 (c.5410-5411delTT; p.1804fsX1819). Homozygosity for this mutation is very likely due to homozygosity by descent from an ancestor common to both parents. This mutation was previously reported in another family from Turkey [1]. No mutation was detected in podocin and WT1 genes. .

During his follow-up, the patient remained oliguric, and peritoneal dialysis was initiated at the 5th week of admission. Subsequently, a fine tremor was observed in his hands despite of normal electrolyte levels. Magnetic resonance imaging scan showed hyperintense lesions in the left thalamus, suggesting a metabolic disease. However, metabolic work-up including tandem mass spectrometry was normal. Lysosomal analysis and analysis of very-longchain fatty acids, phytanic acids, and pristanic acid were negative. Blood lactate level was $2 \mathrm{mmol} / \mathrm{l}$ (normal 0.7 $2 \mathrm{mmol} / \mathrm{l}$ ), and pyruvate was $4.96 \mathrm{mg} / \mathrm{dl}$ (normal $0.3-1 \mathrm{mg}$ / dl). Audiometric analysis revealed sensorineural hearing loss. His electroencephalography was normal, whereas electromyography revealed myopathic findings. A muscle biopsy was performed at 10 months of age to confirm mitochondrial cytopathy due to multiorgan involvement, i.e., steroid-resistant nephrotic syndrome, deafness, and ocular and neurologic findings, as well as electron 
Fig. 1 a Significant mesangial and paramesangial expansion with slightly irregular thickening of glomerular basement membranes [hematoxylin and eosin (H\&E) x400]. b Mesangial expansion and segmental sclerosis were noted in some glomerulus (H\&E $\times 400)$. c, d Shrunken sclerotic glomerulus surrounded by corona of partly hypertrophied vacuolized podocytes (H\&E and Mason trichrome $\mathrm{x} 400$ )
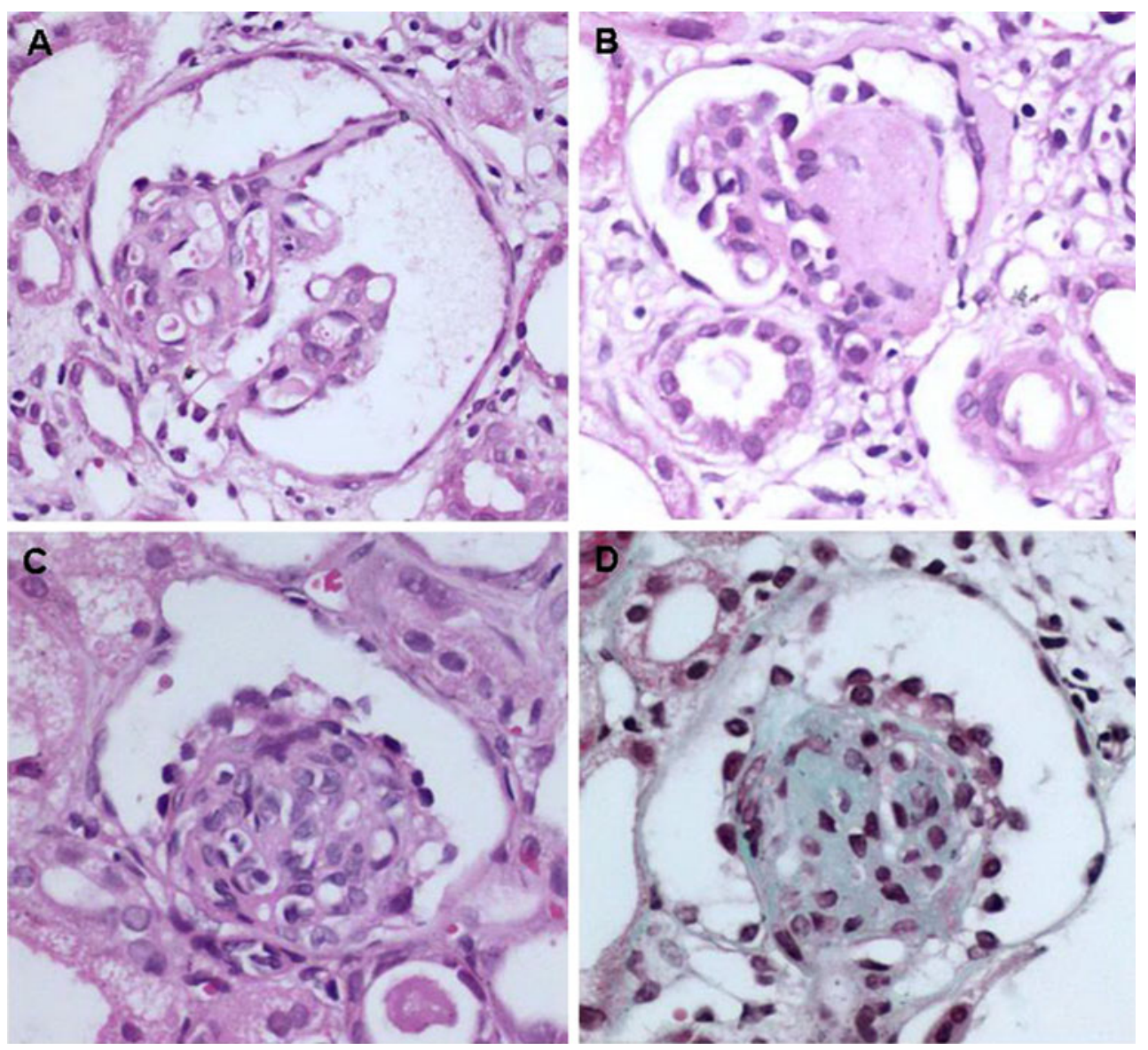

microscopic findings of renal tissue. Although muscle biopsy revealed normal histological findings, the results of biochemical analysis of muscle tissue showed a deficiency of the respiratory chain complex IV (cytochrome C oxidase) (COX) [COX level $58 \mathrm{U} / \mathrm{g}$ noncollagen protein (NCP) (normal 112-351 U/gNCP]. RC complexes I-IV activity was determined in skeletal muscle, as described [7]. Mitochondrial DNA (mtDNA) analysis of muscle excluded the common mitochondrial encephalomyopathy, lactic acidosis, and stroke-like episodes (MELAS) m.3243A $>\mathrm{G}$ and myoclonic epilepsy with ragged-red fibers (MERRF) m.8344A $>\mathrm{G}$ mutations.

After diagnosis, the patient was treated with coenzyme Q10 (CoQ10) for 6 months. During this time, an impressive improvement in his neurologic findings was observed, and he was discharged with peritoneal dialysis at 11 months of age. He was hospitalized because of peritonitis for 3 weeks at 15 months of age and diarrhea at 17 months. However, 2 months later, at 19 months, he died in his hometown after an episode of a febrile illness probably because of sepsis.

Materials and methods

DNA extraction from the patient's muscle and blood was done according to standard purification protocols (Qiagen,
Hildesheim, Germany). Southern blot to test for mtDNA deletions were performed by standard methods; real-time polymerase chain reaction (PCR) to determine mtDNA copy number was performed as described [8]. The nuclear COX10, COX15, and PLCE1 genes were sequenced by standard methods. Biochemical measurement of RC enzymes in skeletal muscle showed an isolated deficiency of COX. Mitochondrial DNA deletions and depletion were excluded by Southern blot and real-time PCR, respectively. Clinical phenotype and biochemical COX deficiency raised the possibility of mutations in COX10; however, genetic analysis of the COX assembly genes COX10 and COX15 did not reveal a pathogenic mutation.

\section{Discussion}

Here we describe the first reported case of DMS associated with PLCE1 mutation and COX deficiency. DMS is a histologically distinct variant of NS characterized by early onset and progression to ESRD. It can be syndromic or nonsyndromic (i.e. isolated). Its etiology and pathogenesis remain unknown. Recently, recessive mutations in PLCE1 (NPHP3) were identified as a novel cause of DMS [2]. Mutational analysis of the NPHS3/PLCE1 gene was 
consistent with the diagnosis of autosomal recessive NS and can be viewed as the cause of the disease in our patient, who did not respond to treatment and developed end-stage renal failure shortly after the diagnosis. However ocular and neurological findings and deafness prompted us to consider a mitochondrial cytopathy. Although light and electron microscopic examinations of renal tissue revealed DMS, severe and unusual arteriolar changes suggested the presence of a possible mitochondrial cytopathy. We confirmed COX deficiency in muscle tissue. Although it is not possible to link renal histological findings directly to COX deficiency, Goldenberg et al. [3] also reported similar renal histological findings regarding renal vascular tissue in patients with respiratory chain complex (RCC) deficiency. Unfortunately enzymological studies and spectrophotometry of renal biopsy were not possible in our patient. However, analysis of muscle tissue revealed a deficiency of RCC IV. Defects of the mitochondrial respiratory chain are recognized as a major cause of human disease. Most of these patients carry mutations in the mtDNA or in nuclear genes encoding subunits or assembly genes of the respiratory chain. However, secondary decrease of the biochemical activities of RC enzymes has been described in several patients with a primarily nonmitochondrial condition [9], complicating the diagnostic workup of patients with RC deficiencies.

The most common renal manifestation of mitochondrial disease in childhood is Fanconi syndrome, usually detected in Pearson or Kearns-Sayre syndrome caused by a single deletion of mtDNA [6]. Mutations in a variety of nuclear genes encoding mitochondrial proteins have been reported recently in association with both tubulopathy and nephrotic syndrome, including coenzyme Q biosynthesis genes (COQ2, $P D S S 2)$, respiratory-chain enzyme-complex assembly genes (BCS1L, COX10), or mtDNA maintenance genes causing mtDNA depletion $(R R M 2 B)$ [10]. Delayed-onset steroidunresponsive NS with focal segmental glomerulosclerosis (FSGS), mostly ascribed to the 3243 MELAS mutation, has been reported in children and adults $[4,5,11]$. Congenital NS caused by RC deficiency was firstly described by Goldenberg et al. [3]. Diomedi-Camassei et al. [12] recently described a novel mutation in COQ2, which was suggested to cause a primary glomerulopathy with earlyonset glomerular lesions. Glomerular involvement of FSGS in the context of mitochondrial dysfunction has been associated in some cases with unusual hyaline lesions that may represent individual myocyte necrosis in afferent arterioles and small arteries [13]. The observed renal arteriolar lesions could cause glomerular hypertension and hyperperfusion that would lead to glomerular epithelial cell abnormalities, including multinucleation and accumulation of abnormal mitochondria in podocytes [14]. It has been suggested that glomerular epithelial cells are susceptible to accumulation of mutant mtDNA and deficient mitochondrial energy supply, leading to podocyte dysfunction followed by development of FSGS. However, our patient had DMS with proven PLCE1 mutation, which may suggest a different pathophysiology for the additional renal tubular changes.

Mitochondrial abnormalities have been previously observed in renal biopsies of NS, irrespective of the course. Mitochondrial dysfunction and downregulation of mitochondria-encoded RC components have been observed in Finnish NS [15]. Apart from a possible causative role of mitochondria in the disease, this suggests a critical role of mitochondria, the first source of energy in the kidney, in maintaining glomerular permeability barrier [3]. However, identification of a PLCE1 mutation as well as RC deficiency makes the situation more complicated in our case. The clinical presentation of our patient is not typical for primary COX deficiency but shows similarities with patients carrying autosomal recessive mutations in COX10. We could not identify pathogenic mutations in the COX10 (or COX15) gene(s), suggesting that PLCE1 deficiency led to a secondary COX deficiency. However, it is not possible as yet to completely rule out a coexisting primary COX deficiency. Given the first-degree cousin relationship of the parents, which puts the child at risk for recessive disease, it is quite likely that the mitochondriopathy phenotype was caused by another mutation homozygous by descent in a yet unidentified recessive gene.

Acknowledgement $\mathrm{FH}$ is an investigator at the Howard Hughes Medical Institute, a Doris Duke Distinguished Clinical Scientist, and is supported by grants from the National Institutes of Health (R01DK076683, RC1DK086542).

\section{References}

1. Gbadegesin R, Hinkes BG, Hoskins BE, Vlangos CN, Heeringa SF, Liu LC, Ozaltin F, Hashmi S, Ulmer F, Cleper R, Ettenger R, Antignac C, Wiggins RC, Zenker M, Hildebrandt F (2008) Mutations in PLCE1 are a major cause of isolated diffuse mesangial sclerosis (IDMS). Nephrol Dial Transplant 23:12911297

2. Hinkes BG, Wiggins RC, Gbadegesin RA, Vlangos CN, Seelow D, Nürnberg G, Garg P, Verma R, Chaib H, Hoskins BE, Ashraf S, Becker C, Hennies HC, Goyal M, Wharram BL, Schachter AD, Mudumana S, Drummond I, Kerjaschki D, Waldherr R, Dietrich A, Ozaltin F, Bakkaloglu A, Cleper R, Basel-Vanagaite L, Pohl M, Griebel M, Tsygin AN, Soylu A, Müller D, Sorli CS, Bunney TD, Katan M, Liu J, Attanasio M, O'Toole JF, Hasselbacher K, Mucha B, Otto EA, Airik R, Kispert K, Kelley GG, Smrcka AV, Gudermann T, Holzman LB, Nürnberg P, Hildebrandt F (2006) Positional cloning uncovers mutations in PLCE1 responsible for a nephrotic syndrome variant that may be reversible. Nat Genet 38:13971405 
3. Goldenberg A, Ngoc LH, Thouret MC, Cormier-Daire V, Gagnadoux MF, Chretien D, Lefrancois C, Geromel V, Rotig A, Rustin P, Munnich A, Paquis V, Antignac C, Gubler MC, Niaudet P, de Lonlay P, Berard E (2005) Respiratory chain deficiency presenting as congenital nephrotic syndrome. Pediatr Nephrol 20:465-469

4. Hirano M, Konishi K, Arata N, Iyori M, Saruta T, Kuramochi S, Akizuki M (2002) Renal complications in a patient with A to $G$ mutation of mitochondrial DNA at the 3243 position of leucine tRNA. Intern Med 41:113-118

5. Hameed R, Raafat F, Ramani P, Gray G, Roper HP, Milford DV (2001) Mitochondrial cytopathy presenting with focal segmental glomerulosclerosis, hypoparathyroidism, sensorineural deafness, and progressive neurological disease. Postgrad Med 77:523-526

6. Hernandez EM, Garcia-Silva MT, Vara J, Campos Y, Cabello A, Muley R, del Hoyo P, Margin MA, Arenas J (2005) Renal pathology in children with mitochondrial diseases. Pediatr Nephrol 20:1299-1305

7. Fischer JC, Ruitenbeek W, Gabreels FJ, Janssen AJ, Renier WO, Sengers RC, Stadhouders AM, ter Laak HJ, Trijbels JM, Veerkamp JH (1986) A mitochondrial encephalomyopathy: the first case with an established defect at the level of coenzyme Q. Eur J Pediatr 144:441-444

8. Reichenbach J, Schubert R, Horvath R, Petersen J, Fütterer N, Malle E, Stumpf A, Gebhardt BR, Koehl U, Schraven B, Zielen S (2006) Fatal neonatal-onset mitochondrial respiratory chain disease with T cell immunodeficiency. Pediatr Res 60:321-3260

9. de Keyzer Y, Valayannopoulos V, Benoist JF, Batteux F, Lacaille F, Hubert L, Chrétien D, Chadefeaux-Vekemans B, Niaudet P,
Touati G, Munnich A, de Lonlay P (2009) Multiple OXPHOS deficiency in the liver, kidney, heart, and skeletal muscle of patients with methylmalonic aciduria and propionic aciduria. Pediatr Res 66(1):91-95

10. Hall AM, Unwin RJ, Hanna MG, Duchen MR (2008) Renal function and mitochondrial cytopathy (MC): more questions than answers? Q J Med 101:755-766

11. Rötig A, Appelkvist EL, Geromel V, Chretien D, Kadhom N, Edery P, Lebideau M, Dallner G, Munnich A, Ernster L, Rustin P (2000) Quinone-responsive multiple respiratory chain dysfunction due to wide spread coenzyme Q10 defficiency. Lancet 356:391395

12. Diomedi-Camassei F, Di Giandomenico S, Santorelli FM, Caridi G, Piemonte F, Montini G, Ghiggeri GM, Murer L, Barisoni L, Pastore A, Muda AO, Valente ML, Bertini E, Emma F (2007) COQ2 nephropathy: a newly described inherited mitochondriopathy with primary renal involvement. J Am Soc Nephrol 18:27732780

13. Doleris LM, Hill GS, Chedin P, Nochy D, Bellanne-Chantelot C, Hanslik T, Bedrossian J, Caillat-Zucman S, Cahen-Varsaux J, Bariety J (2000) Focal segmental glomerulosclerosis associated with mitochondrial cytopathy. Kidney Int 58:1851-1858

14. Hotta O, Inoue C, Miyabayashi S, Furuta T, Takeuchi A, Taguma Y (2001) Clinical and pathologic features of focal segmental glomerulosclerosis with mitochondrial tRNALeu(UUR) gene mutation. Kidney Int 59:1236-1243

15. Khoshnoodi J, Tryggvason K (2001) Congenital nephrotic syndromes. Curr Opin Genet Dev 11:322-327 